\title{
Daytime Sleep as Compensation for the Effects of Reduced Nocturnal Sleep on the Incidence of Hypertension: A Cohort Study
}

\author{
Feifei Yao' \\ Jing Zhao ${ }^{2}$ \\ Yong Cui ${ }^{3}$ \\ Dandan $\mathrm{Yu}^{4}$ \\ Xiangyu Tang $\mathbb{D}^{5}$
}

'Accreditation Department, Shekou People's Hospital, Shenzhen City, Guangdong Province, 5I8067, People's Republic of China; ${ }^{2}$ Department of Preventive Health, Daqing People's Hospital, Daqing City, Heilongjiang Province, People's Republic of China; ${ }^{3}$ Department of Oncology, Shekou People's Hospital, Shenzhen City, Guangdong Province, 518067, People's Republic of China; ${ }^{4}$ Jiangsu Center for Adverse Drug Reaction Monitoring, Nanjing, 210002, People's Republic of China; ${ }^{5}$ Gastroenterology Department, Shekou People's Hospital, Shenzhen City, Guangdong Province, 5I8067, People's Republic of China
Correspondence: Xiangyu Tang

Gastroenterology Department, Shekou

People's Hospital, Shenzhen City,

Guangdong Province, 518067, People's

Republic of China

Email347535969@qq.com
Purpose: The role of sleep duration in the development of hypertension remains controversial. Little is known about the combined effects of nocturnal and daytime sleep. We assessed the association between total sleep duration and the development of hypertension in middle-aged and elderly Chinese adults.

Patients and Methods: Data were obtained from 3 waves of China Health and Retirement Longitudinal Study surveys. Middle-aged and elderly Chinese initially without hypertension were followed biennially from 2011 to 2015. Sleep duration was self-reported. Hypertension was defined as a systolic and/or diastolic blood pressure $\geq 140 / 90$ ( $\mathrm{mmHg}$ ), the current use of anti-hypertensive medications or self-reported hypertension.

Results: Over 31,392 person-years of follow-up, 2682 of 10,176 participants developed hypertension. The multivariable adjusted hazard ratios (HR) (95\% confidence intervals, 95\% CI) for the development of hypertension between those with 0-30 minutes and $\geq 30$ minutes daytime sleep vs those without daytime sleep were $0.67(0.58,0.77)$, and $0.73(0.59,0.92)$, respectively. The protective role of longer periods of daytime sleep ( $>30$ minutes) varied between different subgroups. Compared with moderate nocturnal sleepers, long nocturnal sleepers (HR: 1.66, 95\% CI=1.25-2.21) had an increased risk of hypertension. Compared with moderate nocturnal sleepers without daytime sleep, HRs $(95 \% \mathrm{CI})$ for hypertension were $0.52(0.45,0.59)$ for short nocturnal sleep plus short daytime sleep and $0.55(0.49,0.62)$ for short nocturnal sleep plus long daytime sleep. People with extremely short (HR: 1.34, 95\% $\mathrm{CI}=1.22-1.48$ ) and long (HR: $1.28,95 \% \mathrm{CI}=1.13-1.44$ ) combined sleep periods had an increased risk of hypertension. Consistent results were also found in subgroups stratified by age and gender.

Conclusion: Both extremely long and short total sleep periods were associated with an increased risk of hypertension. People with short or moderate nocturnal sleep durations, especially short nocturnal sleep duration, can benefit from habitual daytime sleep to prevent hypertension.

Keywords: sleep duration, daytime sleep, hypertension, middle-aged and elderly

\section{Introduction}

The prevalence of hypertension in China has increased dramatically from $23.4 \%$ to 28.6\% between 1991 and 2011. ${ }^{1}$ Hypertension is the most common co-morbidity of heart failure patients, ${ }^{2}$ and is a major contributor to cardiovascular disease and diseases burden around the globe. ${ }^{3}$ Moreover, hypertension is a major and modifiable risk factor for stroke, renal disease and other diseases. ${ }^{4}$ Given the increasing 
prevalence of hypertension and its fatal complications, it is necessary to identify modifiable risk factors for hypertension and discuss potential preventive strategies.

Social, economic and technologic factors may result in lifestyle changes that predispose to hypertension. This includes artificial lighting, which plays a fundamental biological role in training the circadian clock. ${ }^{5}$ There has been a trend towards shorter amounts of sleep in the United States since $2013 .{ }^{6}$ A large proportion of the world's population is at an increased risk of environmentally driven circadian rhythm and sleep disruption, and a minority are also genetically predisposed to these disorders. ${ }^{7}$ The consequences of disrupting circadian system and sleep are profound, and include myriad metabolic ramifications. ${ }^{7}$ Sleep has an important effect on the cardiovascular system, and cardiovascular disease can result from changes in sleep rhythm or duration. ${ }^{8}$

Several previous cross-sectional studies have concluded that sleep plays a role in the prevalence of hypertension. ${ }^{9,10}$ However, this association is controversial. Only a few longitudinal studies on this topic were performed in China. One that used data from the China Health and Nutrition Survey found that a short sleep duration was an independent risk factor for hypertension in Chinese adults, whereas a long sleep duration was not. ${ }^{11}$ Another cohort study conducted in Henan province suggested that longer amounts of sleep and a going to sleep late were associated with higher odds of hypertension. ${ }^{12}$ A large sample size longitudinal study whose participants were retired from the Dongfeng Motor Corporation found no significant association between any category of sleep duration or sleep quality and hypertension. ${ }^{13}$

Combining daytime and nighttime sleep into a total sleep pattern is linked to health-relevant outcomes. ${ }^{14}$ However, little is known on the combined effects of nocturnal and daytime sleep duration affected the incidence of hypertension. We therefore retrospectively assessed the relationship between the effects of combined nocturnal and daytime sleep on the risk of hypertension among middle-aged and elderly Chinese adults using prospectively gathered data from the China Health and Retirement Longitudinal Study (CHARLS). We hypothesized that those people who had shorter periods of nocturnal sleep but compensated with daytime sleep (napping) would be at a lower risk of hypertension than those who did not compensate with daytime sleep.

\section{Patients and Methods}

\section{Study Design}

This study utilized 3 waves of CHARLS data which is accessible for free to researchers around the world, from 2011, 2013 and 2015. ${ }^{15}$ Participants were re-assessed every 2 years with a face-to-face and computer-aided personal interview. A 4-stage random sampling method was used to select participants. In the first stage, 150 county-level units from 28 provinces (Excluding Tibet, Taiwan, Hong Kong and Macau) were randomly selected using a probability proportional to size (PPS) sampling technique. All counties were stratified by region, rural/ urban status and per capita statistics on gross domestic product. In the second stage, 3 primary sampling units (PSUs) were selected from each county via PPS sampling. In the third stage, all of the households in each PSU were mapped, and 24 households were randomly selected. Finally, for each selected household, one resident aged $\geq 45$ years was randomly selected to participate in the survey. Physical measurements are made at every 2-year follow-up, and blood sample collection is done once in every two follow-up periods, details on the CHARLS survey have been reported elsewhere. ${ }^{16}$ Ethics approval was not applicable for this study because it was an analysis of previously collected data. In the original survey, ethical approval for all the CHARLS waves was granted from the Institutional Review Board at Peking University. The IRB approval number for the main household survey, including anthropometrics, is IRB00001052-11015; the IRB approval number for biomarker collection was IRB00001052-11014. During the fieldwork, each respondent who agreed to participate in the survey was asked to sign the informed consent. Four separate consents were obtained: one for the main fieldwork, one for the nonblood biomarkers and one for the taking of the blood samples, and another for storage of blood for future analyses. ${ }^{17}$

\section{Study Participants}

Study participants aged 45 years and above and included complete blood pressure and nocturnal/daytime sleep data. We excluded subjects with hypertension, cancer, depression, active treatment for emotional/psychiatric problems, who had ever experienced traffic accident or who were pregnant at the time of initial CHARLS data collection. Subjects who had implausible outlying data were also excluded (Figure 1). 
17708 subjects enrolled in 2011 survey

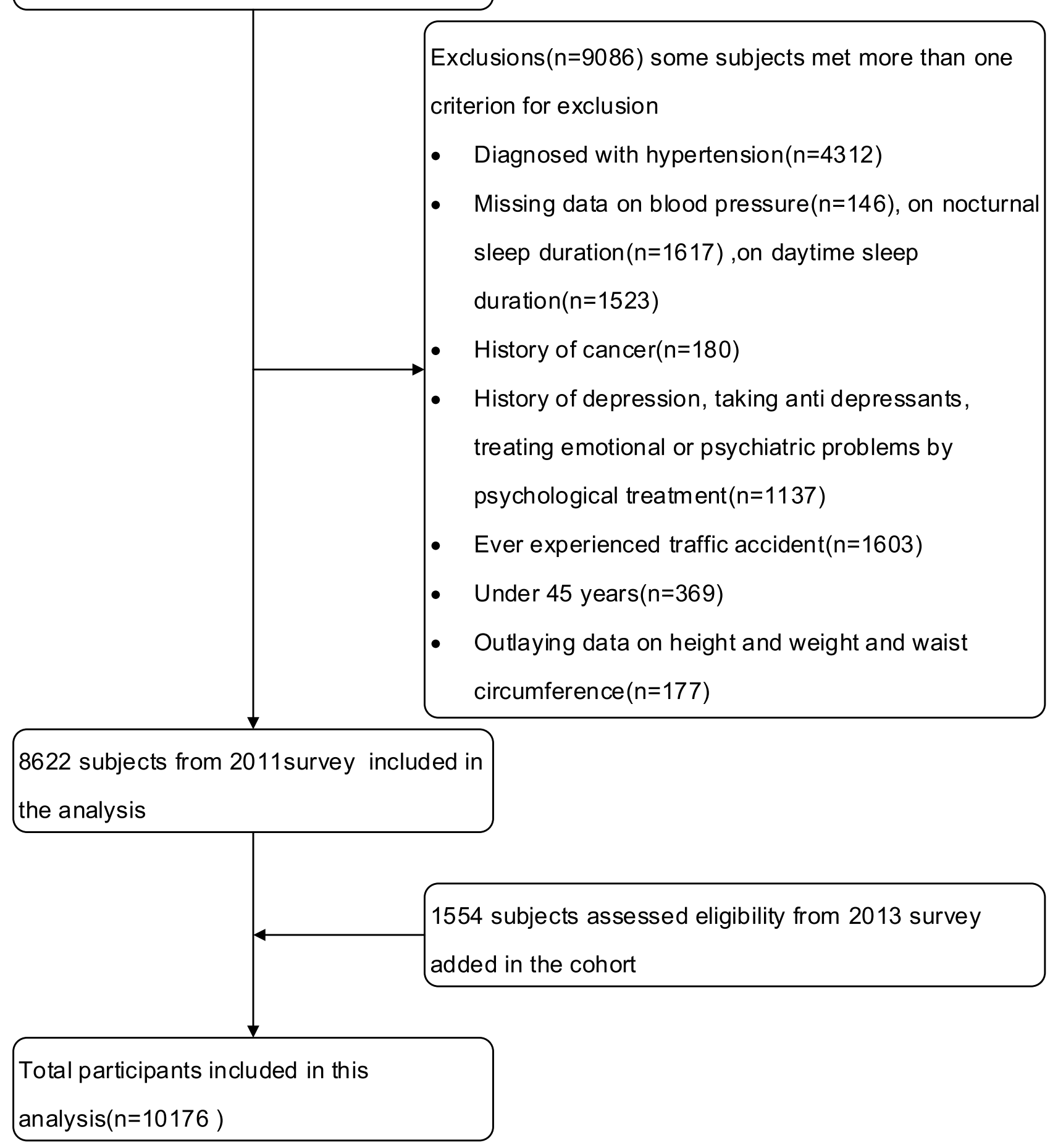

Figure I Sample selection flowchart.

\section{Sleep Duration Assessment}

Nocturnal sleep duration was measured by the question

"During the past month, how many hours of actual sleep did you get at night?". Daytime sleep duration was measured by the question "During the past month, how long did you take a nap after lunch?". In order to better 
represent long-term sleep duration and reduce withinperson variability, the average sleep duration from baseline up to each 2-year follow-up interval was calculated. To reduce the possibility of reverse causation bias, sleep duration after the diagnosis of hypertension was not included, as participants could have changed their sleep duration after developing hypertension. Nocturnal sleep was categorized into 3 groups: short ( $<7$ hours/night), moderate ( $\geq 7$ hours and $\leq 9$ hours) and long $(>9$ hours). ${ }^{18,19}$ Daytime sleep was categorized into 3 groups: 0 hours, $>0$ minutes and $\leq 30$ minutes and $>30$ minutes. The average total sleep period (the sum of nocturnal sleep and daytime sleep duration) was categorized into 4 groups: extremely short ( $\leq 5$ hours/night), moderately short ( $>5$ hours and $\leq 7$ hours), moderate ( $>7$ hours and $\leq 9$ hours) and long ( $>9$ hours).

\section{Outcome Measurement}

The primary outcome of this study was the diagnosis of hypertension. Subjects were followed from the baseline to the visit of hypertension diagnosis or to the last available visit before 2015, whichever came first. Blood pressure was measured for 3 times at 45 -second intervals by trained stuffs using calibrated automated manometers (Omron HEM-7200 Monitor) with subjects in a chair at rest and arm at the same height as their heart. Average of 3 readings of systolic (SBP) and diastolic blood pressure (DBP) was calculated to evaluate hypertension status. Hypertension was defined as systolic blood pressure and/ or diastolic blood pressure $\geq 140 / 90$ ( $\mathrm{mmHg}$ ), current use of anti-hypertensive medications or a self-reported hypertension. $^{20}$

Data of death was obtained from the CHARLS exit and verbal autopsy questionnaire. Participant cause of death was reported by their next of kin or friends.

\section{Assessment of Covariates}

Weight was measured to the nearest $0.1 \mathrm{~kg}$ with a calibrated weight scale (OmronTMHN-286 Scale, Krill Technology, and Yangzhou, China). Height was measured without shoes to the nearest $0.1 \mathrm{~cm}$ with a stadiometer (SecaTM213 Stadiometer, ChinaSeca, Hangzhou, China). ${ }^{21}$ Waist circumference (WC) was measured with soft measuring tape around the participant's waist at the level of the navel at the end of exhalation. Participants wore light clothes when anthropometric variables were measured. $^{21}$ Weight, height and WC were measured once. Based on the recommendations from the World Health Organization (WHO) for Chinese people, body mass index (BMI) was categorized as follows: underweight $<18.5$, normal weight $18.5-23.0$, overweight 23.0 -27 and obese: $\geq 27.5 .^{22}$ Abdominal obesity was defined as a $\mathrm{WC} \geq 80 \mathrm{~cm}$ for women and $\geq 90 \mathrm{~cm}$ for men according to International Diabetes Federation cutoffs for Chinese adults. ${ }^{20}$ All measurements were obtained by trained personnel using standardized procedures.

Self-reporting questionnaires recorded several potential confounders of any relationship between sleep and hypertension. Age (the year they involved in this survey deduct the participants' birth date) was divided into middle-age (45-60 years old) and elderly (60 years of age and above). ${ }^{23}$ Sex was defined as male or female. A positive smoking history was defined as smoking over 100 cigarettes over the participant's lifetime. Smoking status was further divided into 3 groups: never smoked, ever smoked, and currently smoking. Active alcohol intake was defined as $\geq 1$ drink per month. Drinking history was divided into 3 groups: never drank, previously drank and currently drinking. Marital status (married and single), education level (illiterate, primary, secondary and college or higher), job status (the last main job respondents had, divided into agriculture worker, employed, retired and other) and household registration (Hokou, categorized as agricultural Hukou and non-Agricultural Hukou) were also collected. Medical insurance was divided into 2 groups: having medical insurance and no medical insurance. Physical function (Impaired physical function was defined as the inability to independently perform any of the activities described in the questionnaire) and depression symptoms according to the Center for Epidemiological Studies Depression Scale (a score above 10 was defined as significant depression symptoms) ${ }^{21}$ were used to assess depression state. Car possession and the availability of an elevator at home were also evaluated. Participants with a blood glucose concentration $\geq 126 \mathrm{mg} / \mathrm{dL}$ or currently taking anti-diabetic drugs were defined as diabetic.

\section{Statistical Analysis}

We examined the distribution between missing data and observed data across demographic variables, and there was no statistical significance. We therefore assumed that missing data was completely at random, and the multiple imputation method was used to fill-in missing data. 
Data were written as median and quartile for nonnormally distributed continuous variables and as numbers (percentage) for categorical variables. Differences in baseline characteristics were examined using the Mann-Whitney $\mathrm{U}$ and chi-square tests depending on the distribution of the data. Age was used as a continuous variable during data analysis. A multivariate adjusted Cox proportional hazard model was used to estimate the relationship between sleep duration and hypertension. All models adjusted for death to correct for competing risks. All Cox regression models satisfied the proportional hazard assumption. We estimated adjusted hazard ratios (HRs) with $95 \%$ confidence intervals (CIs) for the incidence of hypertension. In model 1, age, sex, job status, household registration, insurance, education, marital status, smoking, drinking, general obesity, abdominal obesity, diabetics, physical function, depression status, housing status and automobile possession were considered. In model 2, we adjusted for all of the factors in model 1 plus the daytime/nocturnal sleep duration and interaction between daytime and nocturnal sleep duration. We performed subgroup analyses based on age (45-60 years vs $\geq 60$ years) and sex (male vs female). Owing to the very small number of participants that had a long duration of both nocturnal and daytime sleep, we combined participants with long nocturnal sleep duration plus 0-30 minutes daytime sleep with those who had a long nocturnal sleep duration plus more than 30 minutes of daytime sleep in our combined effects analysis. The combined effects of night and daytime sleep duration were divided into 8 groups: moderate night sleep + no daytime sleep, short night sleep +30 minutes daytime sleep, short night sleep + more than 30 minutes daytime sleep, short night sleep + no daytime sleep, moderate night sleep +30 minutes daytime sleep, moderate night sleep + more than 30 minutes of daytime sleep, long night sleep + no daytime sleep and long night sleep + more than 30 minutes of daytime sleep. People with 7-9 hours of nocturnal sleep, without daytime sleep, moderate nocturnal sleep without daytime sleep and 7-9 hours of total sleep (sleep duration for 24 hours) were reference groups in all Cox model regressions. All analyses were done with R 3.02 (R-project, Vienna, Austria). Two tailed p-values $<0.05$ were considered statistically significant.

\section{Results}

\section{Baseline Participant Characteristics}

Our cohort included 10,176 participants. Over 31,392 person-years of follow-up (median, 4 years), 2682 participants developed hypertension (incidence rate of: 85.44 per 1000 person-years). There are 394 deaths from other diseases or trauma during the follow-up period.

Compared with those who did not develop hypertension, participants who went on to develop hypertension were older, agricultural, illiterate, single, currently smoking or ever smoked, ever drank, obese, had impaired physical function, lived in a house with an elevator and reported a long nocturnal sleep duration without daytime sleep. Employees were less likely to develop hypertension. Detailed results are shown in Table 1.

\section{Sleep Duration and Combination Effects on the Incidence of Hypertension}

HRs and 95\% CIs for the risk of hypertension across categories of sleep duration and different combinations of sleep duration are shown in Table 2. Compared with those without habitual daytime sleep, people who reported 0-30 minutes and 30 minutes and above of daytime sleep were each independently associated with a reduced incidence of hypertension of 33\% (HR: $0.67,95 \% \mathrm{CI}=0.58$ 0.77 ) and $27 \%$ (HR: $0.73,95 \% \mathrm{CI}=0.59-0.92$ ), respectively. Compared with those who slept 7-9 hours per night, participants who slept less than 7 hours had a $15 \%$ reduced risk of hypertension (HR: $0.85,95 \% \mathrm{CI}=0.80$ 0.92). However, after adjusting for daytime sleep duration and the interaction between nocturnal and daytime sleep, a short nocturnal sleep duration had no significant effect on incidence of hypertension, while those slept more than 9 hours per night (HR: 1.66, 95\% CI=1.25-2.21) had an increased risk of hypertension independently. Compared with those who slept 7-9 hours per day, participants who slept less than 5 hours (HR: 1.34, 95\% CI $=1.22-1.48$ ) and more than 9 hours (HR: $1.28,95 \% \mathrm{CI}=1.13-1.44$ ) had an increased risk of hypertension. The multivariable-adjusted HRs (95\% CIs) for hypertension between those with a short nocturnal sleep duration plus a short daytime sleep period or a short nocturnal sleep period plus a long period of daytime sleep to those who reported a moderate nocturnal sleep period without daytime sleep were 0.52 $(0.45,0.59)$ and $0.55(0.49,0.62)$, respectfully. Similar results were also noted in the combined effects of a moderate nocturnal sleep period and daytime sleep. No statistically significant differences were detected in any category in the setting of a long nocturnal sleep period and additional daytime sleep. 
Table I Baseline Characteristics

\begin{tabular}{|c|c|c|c|c|}
\hline \multirow[t]{2}{*}{ Factors } & \multicolumn{2}{|c|}{ Hypertension } & \multirow[t]{2}{*}{$x^{2} / Z$} & \multirow[t]{2}{*}{$P$} \\
\hline & No (7494) & Yes (2682) & & \\
\hline $\mathrm{Age}^{\mathrm{a}}$ & $56.0(49.0,62.0)$ & $60.0(53.0,68.0)$ & -19.26 & $<0.001$ \\
\hline Sex & & & 1.22 & 0.269 \\
\hline Male & 3545 (73.1\%) & 1302 (26.9\%) & & \\
\hline Female & 3949 (74.1\%) & 1380 (25.9\%) & & \\
\hline Household registration & & & 9.49 & 0.002 \\
\hline Agricultural Hukou & $6093(73.0 \%)$ & $2252(27.0 \%)$ & & \\
\hline Non-Agricultural Hukou & $|40|$ (76.5\%) & $430(23.5 \%)$ & & \\
\hline Job status & & & 52.24 & $<0.001$ \\
\hline Agriculture work & $358 \mid(74.0 \%)$ & $|26|(26.0 \%)$ & & \\
\hline Employees & 972 (8I.3\%) & 224 (I8.7\%) & & \\
\hline Retirement & $693(72.9 \%)$ & $258(27.1 \%)$ & & \\
\hline Others & 2248 (70.5\%) & 939 (29.5\%) & & \\
\hline Insurance & & & 1.65 & 0.122 \\
\hline Yes & $6970(73.5 \%)$ & $2514(26.5 \%)$ & & \\
\hline No & 524 (75.7\%) & $168(24.3 \%)$ & & \\
\hline Education & & & 80.40 & $<0.001$ \\
\hline Illiterate & $1819(68.0 \%)$ & $855(32.0 \%)$ & & \\
\hline Primary & $3048(73.7 \%)$ & $1087(26.3 \%)$ & & \\
\hline Advanced & $2432(77.6 \%)$ & $70 \mathrm{I}(22.4 \%)$ & & \\
\hline College and above & 195 (83.3\%) & $39(16.7 \%)$ & & \\
\hline Marital status & & & 40.95 & $<0.001$ \\
\hline Married & $6728(74.6 \%)$ & $2285(25.4 \%)$ & & \\
\hline Single & 766 (65.9\%) & 397 (34.1\%) & & \\
\hline Smoking status & & & 9.30 & 0.010 \\
\hline Currently smoking & 2369 (73.2\%) & $869(26.8 \%)$ & & \\
\hline Ever smoked & 534 (69.4\%) & 235 (30.6\%) & & \\
\hline Never smoke & $459 I$ (74.4\%) & 1578 (25.6\%) & & \\
\hline Drinking status & & & 14.04 & 0.001 \\
\hline Currently drinking & $|88|$ (72.3\%) & $722(27.7 \%)$ & & \\
\hline Ever drunk & 351 (68.0\%) & 165 (32.0\%) & & \\
\hline Never drink & $5262(73.6 \%)$ & 1795 (26.4\%) & & \\
\hline General obesity & & & 35.25 & $<0.001$ \\
\hline Normal weight & 3456 (76.2\%) & 1078 (23.8\%) & & \\
\hline Underweight & 621 (74.6\%) & $211(25.4 \%)$ & & \\
\hline Over weight & 2586 (71.6\%) & 1025 (28.4\%) & & \\
\hline Obesity & 831 (69.3\%) & 368 (30.7\%) & & \\
\hline Abdominal obesity & & & 99.84 & $<0.001$ \\
\hline Yes & 4403 (70.2\%) & $1869(29.8 \%)$ & & \\
\hline No & 3091 (79.2\%) & $813(20.8 \%)$ & & \\
\hline Diabetics & & & 0.68 & 0.410 \\
\hline Yes & 270 (7I.8\%) & $106(28.2 \%)$ & & \\
\hline No & 7224 (73.7\%) & $2576(26.4 \%)$ & & \\
\hline
\end{tabular}

(Continued) 
Table I (Continued).

\begin{tabular}{|c|c|c|c|c|}
\hline \multirow[t]{2}{*}{ Factors } & \multicolumn{2}{|c|}{ Hypertension } & \multirow[t]{2}{*}{$x^{2} / z$} & \multirow[t]{2}{*}{$P$} \\
\hline & No (7494) & Yes (2682) & & \\
\hline Physical function & & & 61.14 & $<0.001$ \\
\hline Yes & $2222(68.6 \%)$ & 1015 (31.4\%) & & \\
\hline No & $5272(76.0 \%)$ & 1667 (24.0\%) & & \\
\hline Depression status & & & 2.17 & 0.141 \\
\hline Yes & $2774(74.5 \%)$ & $950(25.5 \%)$ & & \\
\hline No & 4720 (73.2\%) & 1732 (26.8\%) & & \\
\hline Housing status & & & 30.68 & $<0.001$ \\
\hline Having elevator & 5771 (72.4\%) & $2203(27.6 \%)$ & & \\
\hline No elevator & 1723 (78.2\%) & 479 (21.8\%) & & \\
\hline Automobile possession & & & 5.89 & 0.015 \\
\hline Yes & 146 (8I.6\%) & 33 (18.4\%) & & \\
\hline No & $7348(73.5 \%)$ & $2649(26.5 \%)$ & & \\
\hline $\mathrm{BMI}^{\mathrm{a}}$ & $22.8(20.5,25.3)$ & $23.2(23.5,25.9)$ & -5.85 & $<0.001$ \\
\hline Waist Measurement & $82.8(76.2,89.6)$ & $86.0(78.2,92.1)$ & -10.77 & $<0.001$ \\
\hline Nocturnal sleep duration & & & 36.79 & $<0.001$ \\
\hline 7-9 hours & 3041 (72.2\%) & II7I (27.8\%) & & \\
\hline$<7$ hour & 4297 (75.3\%) & I 408 (24.7\%) & & \\
\hline$>9$ hours & $156(60.2 \%)$ & $103(39.8 \%)$ & & \\
\hline Daytime sleep duration & & & 98.82 & $<0.001$ \\
\hline 0 minute & $2430(67.8 \%)$ & II 55 (32.2\%) & & \\
\hline $0-30$ minutes & $1829(77.5 \%)$ & $531(22.5 \%)$ & & \\
\hline$>30$ minutes & $3235(76.5 \%)$ & $996(23.5 \%)$ & & \\
\hline Total sleep duration & & & 50.97 & $<0.001$ \\
\hline 7-9 hours & $2884(75.3 \%)$ & $947(24.7 \%)$ & & \\
\hline$\leq 5$ hours & 1167 (68.3\%) & $54 I(31.7 \%)$ & & \\
\hline $5-7$ hours & $2850(75.6 \%)$ & $918(24.4 \%)$ & & \\
\hline$>9$ hours & $593(68.2 \%)$ & $276(31.8 \%)$ & & \\
\hline Combined effects & & & 150.47 & $<0.001$ \\
\hline Moderate night sleep+no daytime sleep & $965(68.4 \%)$ & $446(31.6 \%)$ & & \\
\hline Short night sleep +30 minutes daytime sleep & 1117 (80.8\%) & 265 (19.2\%) & & \\
\hline Short night sleep+more than 30 minutes daytime sleep & 1777 (78.9\%) & $476(21.1 \%)$ & & \\
\hline Short night sleep +no daytime sleep & $1403(67.8 \%)$ & $667(32.2 \%)$ & & \\
\hline Moderate night sleep +30 minutes daytime sleep & $684(73.0 \%)$ & $253(27.0 \%)$ & & \\
\hline Moderate night sleep +more than 30 minutes daytime sleep & 1392 (74.7\%) & $472(25.3 \%)$ & & \\
\hline Long night sleep+no daytime sleep & $62(59.6 \%)$ & $42(40.4 \%)$ & & \\
\hline Long night sleep+more than 30 minutes daytime sleep & $94(60.6 \%)$ & $6 \mathrm{I}(39.4 \%)$ & & \\
\hline Death & & & 11.32 & 0.001 \\
\hline Yes & 319 (81.0\%) & 75 (19.0\%) & & \\
\hline No & 7175 (73.3\%) & 2607 (26.7\%) & & \\
\hline
\end{tabular}

Note: ${ }^{a}$ This variable was analyzed using Mann-Whitney U-test.

\section{Subgroup Analysis}

A protective and significant association between short daytime sleep duration and the incidence of hypertension was observed in males (HR: 0.71, 95\% CI $=0.56-0.87$ ), females (HR: $0.64,95 \% \mathrm{CI}=0.52-0.78$ ), middle-aged (HR: $0.67,95 \% \mathrm{CI}=0.54-0.83$ ) and elderly (HR: 0.67 , 
Table 2 Effects of Sleep Duration on the Incidence of Hypertension

\begin{tabular}{|c|c|c|c|c|c|c|c|c|c|}
\hline \multirow[t]{2}{*}{ Factors } & \multicolumn{3}{|c|}{ Crude } & \multicolumn{3}{|c|}{ Adjusted Model I } & \multicolumn{3}{|c|}{ Adjusted Model 2} \\
\hline & HR & $\begin{array}{l}\text { HR 95\% } \\
\text { Cl }\end{array}$ & $\mathbf{P}$ & HR & $\begin{array}{l}\text { HR 95\% } \\
\text { Cl }\end{array}$ & $\mathbf{P}$ & HR & $\begin{array}{l}\text { HR 95\% } \\
\text { Cl }\end{array}$ & $\mathbf{P}$ \\
\hline \multicolumn{10}{|l|}{ Daytime sleep duration } \\
\hline 0 minute & Reference & Reference & & Reference & Reference & & Reference & Reference & \\
\hline $0-30$ minutes & 0.61 & $0.56,0.68$ & $<0.001$ & 0.62 & $0.56,0.68$ & $<0.001$ & 0.67 & $0.58,0.77$ & $<0.001$ \\
\hline$>30$ minutes & 0.67 & $0.62,0.72$ & $<0.001$ & 0.64 & $0.59,0.69$ & $<0.001$ & 0.73 & $0.59,0.92$ & 0.006 \\
\hline \multicolumn{10}{|l|}{ Nocturnal sleep duration } \\
\hline 7-9 hours & Reference & Reference & & Reference & Reference & & Reference & Reference & \\
\hline$<7$ hours & 0.86 & $0.80,0.92$ & $<0.001$ & 0.85 & $0.80,0.92$ & $<0.001$ & 0.93 & $0.80,1.07$ & 0.290 \\
\hline$>9$ hours & 1.56 & $1.32,1.85$ & $<0.001$ & 1.41 & $1.19,1.67$ & $<0.001$ & 1.66 & $1.25,2.21$ & $<0.001$ \\
\hline \multicolumn{10}{|l|}{ Total sleep duration } \\
\hline 7-9 hours & Reference & Reference & & Reference & Reference & & & & \\
\hline$\leq 5$ hours & 1.38 & $1.26,1.52$ & $<0.001$ & 1.34 & $1.22,1.48$ & $<0.001$ & & & \\
\hline $5-7$ hours & 0.99 & $0.92,1.08$ & 0.880 & 1.02 & $0.94,1.10$ & 0.680 & & & \\
\hline$>9$ hours & 1.37 & $1.22,1.55$ & $<0.001$ & 1.28 & $1.13,1.44$ & $<0.001$ & & & \\
\hline \multicolumn{10}{|l|}{ Combined effects } \\
\hline $\begin{array}{l}\text { Moderate night sleep+no daytime } \\
\text { sleep }\end{array}$ & Reference & Reference & & Reference & Reference & & & & \\
\hline $\begin{array}{l}\text { Short night sleep }+30 \text { minutes } \\
\text { daytime sleep }\end{array}$ & 0.52 & $0.45,0.59$ & $<0.001$ & 0.52 & $0.45,0.59$ & $<0.001$ & & & \\
\hline $\begin{array}{l}\text { Short night sleep+more than } 30 \\
\text { minutes daytime sleep }\end{array}$ & 0.59 & $0.52,0.66$ & $<0.001$ & 0.55 & $0.49,0.62$ & $<0.001$ & & & \\
\hline Short night sleep +no daytime sleep & 0.99 & $0.89,1.10$ & 0.860 & 0.97 & $0.88,1.08$ & 0.610 & & & \\
\hline $\begin{array}{l}\text { Moderate night sleep }+30 \text { minutes } \\
\text { daytime sleep }\end{array}$ & 0.76 & $0.66,0.87$ & $<0.001$ & 0.74 & $0.65,0.85$ & $<0.001$ & & & \\
\hline $\begin{array}{l}\text { Moderate night sleep +more than } \\
30 \text { minutes daytime sleep }\end{array}$ & 0.74 & $0.65,0.83$ & $<0.001$ & 0.70 & $0.62,0.78$ & $<0.001$ & & & \\
\hline Long night sleep+no daytime sleep & 1.37 & $1.05,1.78$ & 0.020 & 1.27 & $0.98,1.64$ & 0.070 & & & \\
\hline $\begin{array}{l}\text { Long night sleep+more than } 30 \\
\text { minutes daytime sleep }\end{array}$ & 1.24 & $0.99,1.57$ & 0.070 & 1.07 & $0.84,1.34$ & 0.600 & & & \\
\hline
\end{tabular}

95\% $\mathrm{CI}=0.56-0.81$ ) (Figures 2-5), while a long daytime sleep duration (>30 minutes) did not impart a significant protective effect on the incidence of hypertension in females and middle-aged participants (Figures 3 and 4).
The negative effects of long nocturnal sleep duration on the incidence of hypertension were found in females (HR: 1.89, 95\% CI=1.28-2.81) and middle-aged (HR: 2.20, 95\% CI=1.42-3.40) participants (Figures 3,4 and 5). 


\section{Factors}

Daytime sleep duration**

Ominute

$0-30$ minutes

$>30$ minutes

Nocturnal sleep duration**

7-9 hours

$<7$ hours

$>9$ hours

Total sleep duration*

7-9 hours

$\leq 5$ hours

5-7 hours

$>9$ hours

Combined effects*

Moderate night sleep+no daytime sleep

Short night sleep+30 minutes daytime sleep

Short night sleep+more than 30 minutes daytime sleep

Short night sleep+no daytime sleep

Moderate night sleep+30 minutes daytime sleep

Moderate night sleep+more than 30 minutes daytime sleep 1028

Long night sleep+no daytime sleep

Long night sleep+more than 30 minutes daytime sleep

\section{$\mathbf{N}$}

$\mathrm{HR}(95 \% \mathrm{Cl})$

1495

1073

2279

$0.71(0.56,0.87)<0.001$

$0.68(0.50,0.87) \quad 0.016$

2094

2628

125

1963

670

1755

459

621

606

1194

828

445

028

46

79
$0.68(0.50,0.87)$

$0.84(0.68,1.04) \quad 0.110$

$1.42(0.93,2.16) \quad 0.110$

$1.28(1.11,1.48) \quad<0.001$

$0.92(0.82,1.03) \quad 0.140$

$1.16(0.99,1.37) \quad 0.070$

$0.55(0.45,0.68)<0.001$

$0.60(0.51,0.70)<0.001$

$0.96(0.81,1.13) \quad 0.600$

$0.89(0.74,1.07) \quad 0.200$

$0.71(0.60,0.83) \quad<0.001$

$1.30(0.90,1.87) \quad 0.160$

$1.11(0.80,1.53) \quad 0.530$

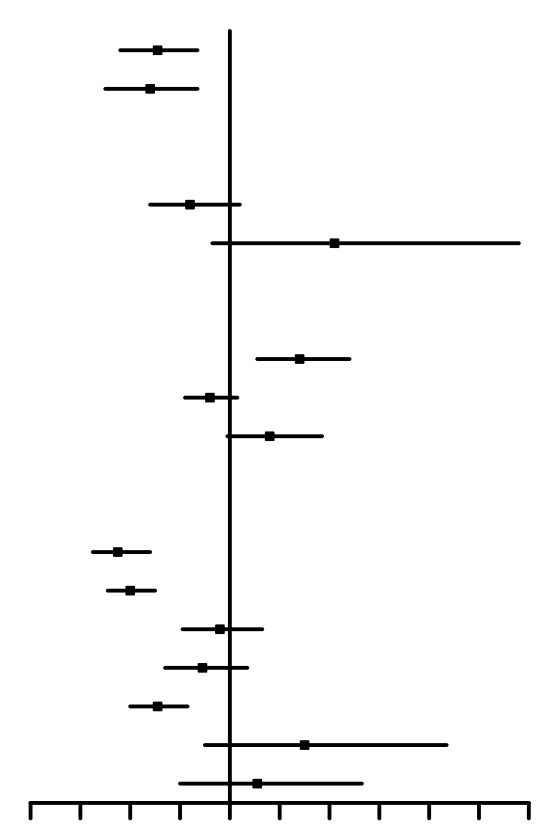

$\begin{array}{lllllllllll}0.2 & 0.4 & 0.6 & 0.8 & 1 & 1.2 & 1.4 & 1.6 & 1.8 & 2 & 2.2\end{array}$

Figure 2 The effects of sleep duration on the incidence of hypertension in males.

Notes: *Adjusted factors in model I (age, sex, job status, neighborhood registration, insurance, education, marital status, smoking, drinking, general obesity, abdominal obesity, diabetes, physical function, depression status, housing status and automobile possession). **Adjusted factors in model 2 (factors in model I plus the interaction between daytime and nocturnal sleep and daytime/ nocturnal sleep duration).

The negative effects of extremely short and long total sleep on the incidence of hypertension were broadly similar between groups classified by age and sex (Figures 2-5).

In the combination effects analysis, a protective association between moderate nocturnal sleep duration plus 0-30 minutes of daytime sleep and the incidence of hypertension was in females (HR: $0.62,95 \% \mathrm{CI}=0.51-$ 0.76 ), middle-aged (HR: $0.72,95 \% \mathrm{CI}=0.59-0.87$ ) and elderly (HR: $0.80,95 \% \mathrm{CI}=0.67-0.96$ ) participants but not in males (HR: 0.89, 95\% CI=0.74-1.07). For males, compared with a moderate nocturnal sleep duration without daytime sleep, people with a short sleep duration plus a short (HR: $0.55,95 \% \mathrm{CI}=0.45-0.68$ ) or long (HR: $0.60,95 \% \mathrm{CI}=0.51-0.70$ ) period of daytime sleep had a reduced risk of hypertension. Similar results were also noted in the combined effects of moderate nocturnal sleep and $>30$ minutes of daytime sleep (HR: 0.71, 95\% $\mathrm{CI}=0.60-0.83)$. Participants with adequate nocturnal sleep duration had a slightly weaker protective benefit of daytime sleep than those with short nocturnal sleep duration. No statistically significant difference was found in any sub-categories of participants with a long nocturnal sleep duration and daytime sleep. Results are shown in Figure 2. The effects of sleep duration on the incidence of hypertension were broadly similar between age and sex subgroups (Figures 2-5).

\section{Discussion}

In this retrospective analysis of a prospectively acquired middle-aged and elderly cohort, participants who reported routine daytime sleep periods were less likely develop hypertension. The protective role of a longer duration of daytime sleep on hypertension varied between sex and age subgroups. The hazard effects of long nocturnal sleep duration on hypertension were only found in females and middle-aged participants. Participants with extremely short or long total sleep periods tended to develop hypertension. Participants with a short or moderate nocturnal sleep plus daytime sleep had a significantly reduced incidence of hypertension. These results were also observed in subgroups analyses stratified by age and sex. To the best of our 
Factors

\section{Daytime sleep duration ** \\ Ominute \\ 0-30 minutes \\ $>30$ minutes \\ Nocturnal sleep duration** \\ 7-9 hours \\ $<7$ hours \\ $>9$ hours}

Total sleep duration*

7-9 hours

$\leq 5$ hours

5-7 hours

$>9$ hours

Combined effects*

Moderate night sleep+no daytime sleep

Short night sleep +30 minutes daytime sleep

Short night sleep+more than 30 minutes daytime sleep

Short night sleep+no daytime sleep

Moderate night sleep +30 minutes daytime sleep

Moderate night sleep+more than 30 minutes daytime

sleep Long night sleep+no daytime sleep

Long night sleep+more than 30 minutes daytime sleep
N $\quad H R(95 \% \mathrm{Cl})$

$\begin{array}{ccc}2090 & & \\ 1287 & 0.64(0.52,0.78) & <0.001 \\ 1952 & 0.79(0.57,1.09) & 0.150 \\ & & \\ 2118 & & \\ 3077 & 0.99(0.82,1.21) & 0.930 \\ 134 & 1.89(1.28,2.81) & 0.002 \\ & & \\ 1868 & & \\ 1038 & 1.40(1.23,1.60) & <0.001 \\ 2013 & 1.13(1.00,1.27) & 0.045 \\ 410 & 1.42(1.19,1.68) & <0.001 \\ & & \\ 790 & & \\ 776 & 0.49(0.41,0.59) & <0.001 \\ 1059 & 0.51(0.44,0.61) & <0.001 \\ 1242 & 0.98(0.85,1.12) & 0.730 \\ 492 & 0.62(0.51,0.76) & <0.001 \\ 836 & 0.71(0.60,0.83) & <0.001 \\ 58 & 1.25(0.88,1.78) & 0.210 \\ 76 & 1.08(0.77,1.51) & 0.650\end{array}$

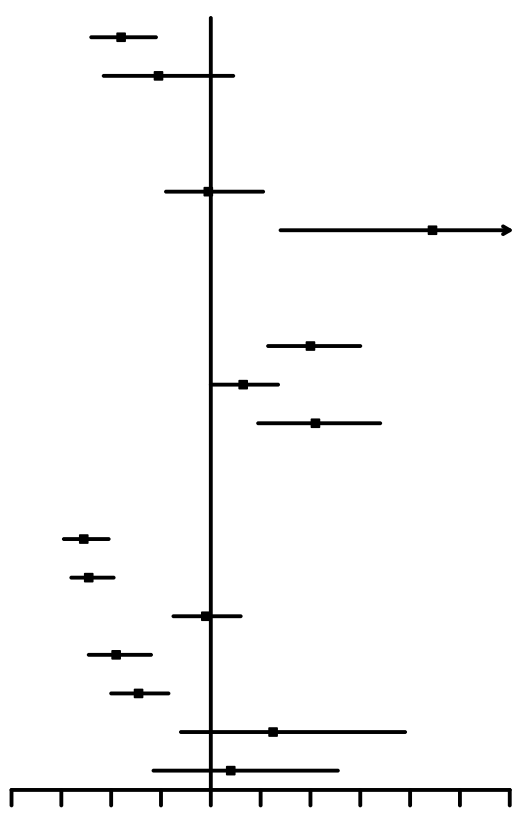

$\begin{array}{llllllllllll}0.2 & 0.4 & 0.6 & 0.8 & 1 & 1.2 & 1.4 & 1.6 & 1.8 & 2 & 2.2\end{array}$

Figure 3 The effects of sleep duration on the incidence of hypertension in females.

Notes: *Adjusted factors in model I (age, sex, job status, neighborhood registration, insurance, education, marital status, smoking, drinking, general obesity, abdominal obesity, diabetes, physical function, depression status, housing status and automobile possession). **Adjusted factors in model 2 (factors in model I plus the interaction between daytime and nocturnal sleep and daytime/ nocturnal sleep duration).

knowledge this is the first analysis of prospective data on the contribution of sleep factors to the development of hypertension used CHARLS data. Although daytime naps are usually related to nocturnal sleep, the great majority of studies assess their effects on health outcomes independently. This study examined patterns of sleep behavior that take into account both nighttime and daytime sleep. Moreover, we analyzed the relationship between sleep duration and the incidence of hypertension by calculating average sleep duration, which reflects long-term sleep status.

The joint consensus statement of the American Academy of Sleep Medicine and Sleep Research Society recommends 7-9 hours of sleep for a healthy adult. ${ }^{18}$ This recommendation is widely used by research studies, including our own. However, we found that people who slept less than 7 hours per night had a reduced risk of hypertension in adjusted model 1 . That the participants in our study were middle-aged and elderly may account for this result, as they do not need as much sleep as young adults and they have more opportunities to take a nap during the daytime. Middle-aged and elderly people are also more likely to report poor sleep quality or a sleep disorder. Naps seem to compensate for poor subjective sleep quality, and to some extent for a short duration of sleep. ${ }^{24}$ It was reported that people in China were $18 \%$ more likely to take a daytime nap $(\mathrm{OR}=1.18, \mathrm{p}<0.01)$ after transitioning to retirement. ${ }^{25}$ Most of the participants in our study were retired at home, and $64.77 \%$ took habitual daytime naps. A prior study found that napping had no negative impact on nighttime sleep quality or duration, resulting in a significant increase in total 24-hour sleep duration among older adults. ${ }^{26}$ We believe that participants' short nocturnal sleep duration or poor sleep quality was compensated for by daytime sleep, thereby reducing adverse health outcomes. Moreover, we found that people had habitual daytime sleep had reduced risk of incidence of hypertension independently. To verify our speculation we further adjusted for daytime sleep duration as well as the interaction between daytime and nighttime sleep duration in model 2. As a result, the adverse effects of short nocturnal sleep duration on the incidence of hypertension disappeared. Additionally, participants who reported extremely short ( $\leq 5$ hours) or long ( $>9$ hours) sleep durations per day had a significantly increased risk of hypertension, while we did not identify a relationship between short total 


\section{Factors}

\section{Daytime sleep duration ** \\ Ominute \\ 0-30 minutes \\ $>30$ minutes}

Nocturnal sleep duration ${ }^{* *}$

7-9 hours

$<7$ hours

$>9$ hours

Total sleep duration*

7-9 hours

$\leq 5$ hours

5-7 hours

$>9$ hours

Combined effects*

Moderate night sleep+no daytime sleep

Short night sleep +30 minutes daytime sleep

Short night sleep+more than 30 minutes daytime sleep

Short night sleep+no daytime sleep

Moderate night sleep +30 minutes daytime sleep

Moderate night sleep+more than 30 minutes daytime

sleep Long night sleep + no daytime sleep

Long night sleep+more than 30 minutes daytime sleep
$\mathbf{N}$

\section{$\mathrm{HR}(95 \% \mathrm{Cl})$}

2198

1467

2546

$0.67(0.54,0.83)<0.001$

$0.87(0.62,1.22) \quad 0.430$

2678

3396

137

$1.02(0.81,1.27)$

$2.20(1.42,3.40)$

0.890

$<0.001$

2471

894

2351

495

$1.40(1.20,1.63) \quad<0.001$

$1.07(0.95,1.21) \quad 0.270$

$1.33(1.10,1.59) \quad 0.003$

917

827

1346

1223

617

1144

58

79

$\begin{array}{lc}0.46(0.38,0.57) & <0.001 \\ 0.55(0.47,0.66) & <0.001 \\ 1.04(0.89,1.21) & 0.660 \\ 0.72(0.59,0.87) & <0.001 \\ 0.75(0.63,0.88) & <0.001 \\ 1.38(0.90,2.10) & 0.140 \\ 1.18(0.79,1.76) & 0.420\end{array}$

$1.18(0.79,1.76) \quad 0.420$

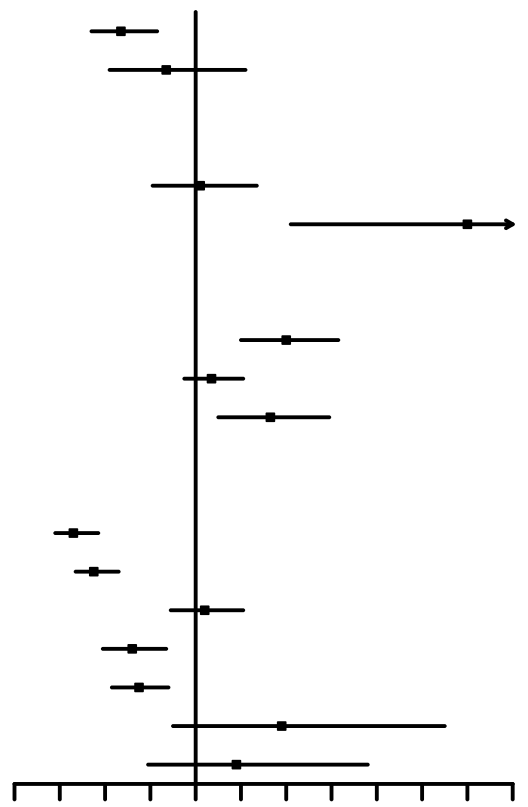

$0.20 .4 \quad 0.60 .8 \quad 1 \quad 1.21 .41 .61 .8 \quad 2 \quad 2.22 .4$

Figure 4 The effects of sleep duration on the incidence of hypertension in middle-aged.

Notes: *Adjusted factors in model I (age, sex, job status, neighborhood registration, insurance, education, marital status, smoking, drinking, general obesity, abdominal obesity, diabetes, physical function, depression status, housing status and automobile possession). **Adjusted factors in model 2 (factors in model I plus the interaction between daytime and nocturnal sleep and daytime/ nocturnal sleep duration).

sleep duration (5-7hours) on the incidence of hypertension. This result was in line with the possibly acceptable hours (5-9 hours/day) recommended by the National Sleep Foundation for older adults ( $\geq 65$ years). ${ }^{27}$ The National Sleep Foundation currently recommends sleeping seven to seventeen hours per 24 hour period, with age-specific recommendations decreasing with increasing age. ${ }^{27}$ The results of our study suggest that nocturnal and daytime sleep duration should not be analyzed separately. We found those with a short or moderate nocturnal sleep duration without a daytime nap did not have any altered risk of hypertension, those with daytime sleep had a reduced risk of developing hypertension. These findings were broadly consistent across sex and age groups. Besides we found that among those had habitual daytime sleep, short nocturnal sleepers had fewer risks of hypertension than moderate sleepers in all subjects and subgroups. Daytime sleep may therefore play more important role in the primary prevention of hypertension in middle-aged and elderly Chinese adults who only sleep at night for short periods. Afternoon napping reverses changes in salivary interleukin-6 and urinary nor epinephrine that are induced by sleep restriction. ${ }^{28}$ This mechanism suggests that napping could improve neuroendocrine stress and immune recovery, with a potential prophylactic long-term effect on cardiovascular health. ${ }^{28}$ This mechanism may also contribute to the favorable effect of an afternoon nap on the risk of hypertension. Our results suggest that middle-aged and elderly individuals, especially those who slept at night for only short periods, may benefit from habitual naps to prevent hypertension as an alternative to prolonged nocturnal sleep.

A large cohort study performed on the Kailuan community in the city of Tangshan, China found that $\leqslant 5 \mathrm{~h}$ of sleep per night was associated with an increased risk of hypertension in females and participants aged $<60$ years. ${ }^{29}$ Another study also performed in China concluded that a short nocturnal sleep duration was a significant risk factor for hypertension in younger subjects, with no association among older subjects. ${ }^{30}$ Both short and long nocturnal sleep periods were found to be significantly associated with hypertension among middle-aged and elderly participants in a cross sectional study, while no significant association was detected in a prospective study. ${ }^{13}$ Our study revealed that a long nocturnal sleep duration was associated with an increased risk of 
Factors

\section{Daytime sleep duration ** Ominute \\ 0-30 minutes \\ $>30$ minutes \\ Nocturnal sleep duration** \\ 7-9 hours \\ $<7$ hours \\ $>9$ hours \\ Total sleep duration* \\ 7-9 hours \\ $\leq 5$ hours \\ $5-7$ hours \\ $>9$ hours}

Combined effects*

Moderate night sleep+no daytime sleep

Short night sleep +30 minutes daytime sleep

Short night sleep+more than 30 minutes daytime sleep

Short night sleep+no daytime sleep

Moderate night sleep +30 minutes daytime sleep

Moderate night sleep+more than30 minutes daytime sleep

Long night sleep+no daytime sleep

Long night sleep+more than 30 minutes daytime sleep
$\mathbf{N}$

1387

893

1685

1534

2309

122

1360

814

1417

374

494

555

907

847

320

720

46

76
$\mathrm{HR}(95 \% \mathrm{Cl})$

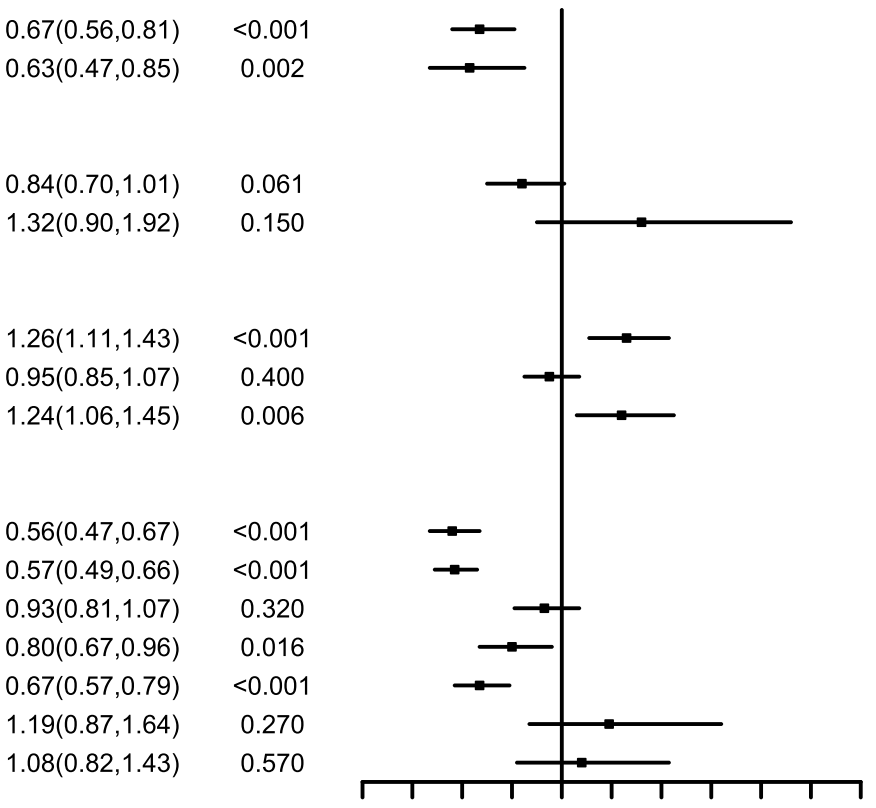

$\begin{array}{llllllllllll}0.2 & 0.4 & 0.6 & 0.8 & 1 & 1.2 & 1.4 & 1.6 & 1.8 & 2 & 2.2\end{array}$

Figure 5 The effects of sleep duration on the incidence of hypertension in elderly.

Notes: *Adjusted factors in model I (age, sex, job status, neighborhood registration, insurance, education, marital status, smoking, drinking, general obesity, abdominal obesity, diabetes, physical function, depression status, housing status and automobile possession). **Adjusted factors in model 2 (factors in model I plus the interaction between daytime and nocturnal sleep and daytime/ nocturnal sleep duration).

hypertension, but no significant association was detected in our combination effects analysis. This may be due to the small sample size that was used in the combination effects analysis, so this finding should be interpreted with caution. Although the aforementioned studies were all conducted in China, their results differed from one another. This is partially explained by the different population and sleep duration categories that were used. A cross sectional study that analyzed the relationship between nocturnal sleep duration and hypertension using CHARLS 2015 data found that a short and long nocturnal sleep duration affected the prevalence of hypertension in middle-aged and elderly adults, with age- and gender-related effects. ${ }^{31}$ Both this study and ours used the CHARLS data, but their results were partially discrepant with ours. The increased risk of hypertension among middle-aged participants who reported long nocturnal sleep duration was in line with our study. What differs from our results is that they also discovered the adverse effects of short-term sleep on hypertension. The discrepancies my explained by the following 2 reasons: different study methods and part of the participants included in study. Another cross-sectional study found that a short amount of sleep per day had a significant adverse effect on the prevalence of hypertension. This result was consistent with the present work, but no adverse effects of long sleep duration were identified in their study. ${ }^{11}$ Sleep duration was based on survey response in most previous studies. However, it varied across the 33year recording period and can fluctuate for a variety of reasons. ${ }^{32}$ As such the effects of sleep on hypertension may change. An analysis of a single point in time may therefore be unable to effectively predict the risk of hypertension. In the present study sleep duration was assessed repeatedly in 3 waves, with the average sleep duration before hypertension was diagnosed used as data points. Although sleep duration was not constantly measured during each interval, average sleep duration may better reflect long-term sleep status. Hypertension is a chronic disease, so to better associate it with sleep duration, future cohort studies should include additional assessment points.

Sleep duration was assessed in the present and prior works via self-reporting. Self-reported total sleep duration 
was overestimated by 60 minutes in Chinese adults compared with wrist actigraphy measurements. ${ }^{33}$ This recall bias may partially account for the discrepant results between studies. Sleep is a complex behavior that involves interdependent daytime naps and nocturnal sleep periods of varying time, length and quality. ${ }^{14}$ Long-term objective measurements are required to better assess sleep in future prospective analyses.

\section{Strengths and Limitations}

The primary strengths of our study are its prospective study design, use of a nationally representative sample and longitudinal measurement of sleep duration. However, it has several limitations. First, a well-designed sleep quality questionnaire was not available. Second, the physical activity level of the majority of survey respondents was missing and therefore could not be taken into account. Due to the different intensity of physical activity required for different jobs, respondent job status was used to compensate for missing activity. Third, sleep duration in CHARLS was self-reported, inviting recall bias. It was previously reported that the agreement in biphasic sleep pattern between self-reported and actigraphy assessments was $70.6 \%$ among Omani people 40 years and older, and overall agreement between measured sleep pattern (including monophasic, biphasic, polyphasic) between the two methods was $57 \% .^{34}$ Fourth, our study is based on a cohort of middle-aged and elderly Chinese adults. The findings from this study may therefore not be generalizable to younger people or people from other countries. Fifth, we did not record when subjects who switched sleep habits during follow-up did so. We could not assess the effect of switch time on hypertension. Sixth, as participant blood pressure was not measured consistently during each follow-up, the exact time of the development of hypertension is unclear. Finally, information on participants' sodium intake and a family history of hypertension are unavailable due to the limitations of CHARLS, so were not considered in this study.

\section{Conclusion}

Middle-aged or elderly Chinese adults with extremely short or long total sleep durations were more likely to develop hypertension. People with insufficient sleep at night who compensated with daytime sleep reduced their risk of hypertension. The beneficial effects of habitual daytime sleep duration on hypertension were more prominent among people with short nocturnal sleep duration than those with moderate nocturnal sleep duration. Given the controversial results of prior studies and the complexity of sleep, long-term objectively measured sleep duration needs to be assessed in future prospective studies.

\section{Acknowledgment}

The authors thank the CHARLS team for providing the data for our study.

\section{Disclosure}

None of the authors have any potential conflicts of interest associated with this research.

This research did not receive any specific grant from funding agencies in the public, commercial, or not-forprofit sectors.

\section{References}

1. Guo J, Zhu YC, Chen YP, Hu Y, Tang XW, Zhang B. The dynamics of hypertension prevalence, awareness, treatment, control and associated factors in Chinese adults: results from CHNS 1991-2011. J Hypertens. 2015;33(8):1688-1696. doi:10.1097/HJH.0000000000000594

2. Saczynski JS, Go AS, Magid DJ, et al. Patterns of comorbidity in older adults with heart failure: the Cardiovascular Research Network PRESERVE study. J Am Geriatr Soc. 2013;61(1):26-33. doi:10.1111/ jgs. 12062

3. Forouzanfar MH, Alexander L, Anderson HR, et al. Global, regional, and national comparative risk assessment of 79 behavioural, environmental and occupational, and metabolic risks or clusters of risks in 188 countries, 1990-2013: a systematic analysis for the Global Burden of Disease Study 2013. Lancet. 2015;386(10010):2287-2323.

4. Hao G, Wang X, Chen Z, et al. Prevalence of heart failure and left ventricular dysfunction in China: the China Hypertension Survey, 20122015. Eur J Heart Fail. 2019;21(11):1329-1337. doi:10.1002/ejhf.1629

5. Ohayon MM, Milesi C. Artificial outdoor night time lights associate with altered sleep behavior in the American General Population. Sleep. 2016;39(6):1311-1320. doi:10.5665/sleep.5860

6. Sheehan CM, Frochen SE, Walsemann KM, Ailshire JA, Are US. adults reporting less sleep?: findings from sleep duration trends in the National Health Interview Survey, 2004-2017. Sleep. 2019;42(2). doi:10.1093/sleep/zsy221

7. Potter GD, Skene DJ, Arendt J, Cade JE, Grant PJ, Hardie LJ. Circadian rhythm and sleep disruption: causes, metabolic consequences, and countermeasures. Endocr Rev. 2016;37(6):584-608.

8. Wang C, Bangdiwala SI, Rangarajan S, et al. Association of estimated sleep duration and naps with mortality and cardiovascular events: a study of 116632 people from 21 countries. Eur Heart J. 2019;40(20):1620-1629. doi:10.1093/eurheartj/ehy695

9. Grandner M, Mullington JM, Hashmi SD, Redeker NS, Watson NF, Morgenthaler TI. Sleep duration and hypertension: analysis of > 700,000 adults by age and sex. J Clin Sleep Med. 2018;14 (06):1031-1039. doi:10.5664/jcsm.7176

10. Sun XM, Yao S, Hu SJ, et al. Short sleep duration is associated with increased risk of pre-hypertension and hypertension in Chinese early middle-aged females. Sleep Breath. 2016;20(4):1355-1362. doi:10.1007/s11325-016-1392-2

11. Feng X, Liu Q, Li Y, Zhao F, Chang H, Lyu J. Longitudinal study of the relationship between sleep duration and hypertension in Chinese adult residents (CHNS 2004-2011). Sleep Med. 2019;58:88-92. doi:10.1016/j.sleep.2019.01.006 
12. Zhang H, Zhao X, Li Y, et al. Night sleep duration and sleep initiation time with hypertension in Chinese rural population: the Henan Rural Cohort. Eur J Public Health. 2020;30(1):164-170. doi:10.1093/eur$\mathrm{pub} / \mathrm{ckz} 142$

13. Wang D, Zhou Y, Guo Y, et al. The effect of sleep duration and sleep quality on hypertension in middle-aged and older Chinese: the Dongfeng-Tongji Cohort Study. Sleep Med. 2017;40:78-83. doi:10.1016/j.sleep.2017.09.024

14. Devine JK, Wolf JM. Integrating nap and night-time sleep into sleep patterns reveals differential links to health-relevant outcomes. J Sleep Res. 2016;25(2):225-233. doi:10.1111/jsr.12369

15. China Health and Retirement Longitudinal Study. Available from: http:/charls.pku.edu.cn/pages/data/111/en.html http://charls.pku.edu. cn/pages/data/111/zh-cn.html.Accessed June 30, 2021

16. Zhao Y, Hu Y, Smith JP, Strauss J, Yang G. Cohort profile: the China Health and Retirement Longitudinal Study (CHARLS). Int J Epidemiol. 2014;43(1):61-68. doi:10.1093/ije/dys203

17. Zhao Y, Strauss J, Chen X, et al. China Health and Retirement Longitudinal Study Wave 4 User's Guide. 2020.National School of Development, Peking University

18. Watson NF, Badr MS, Belenky G, et al. Recommended amount of sleep for a healthy adult: a Joint Consensus Statement of the American Academy of Sleep Medicine and Sleep Research Society. Sleep. 2015;38(6):843-844. doi:10.5665/sleep.4716

19. Mukherjee S, Patel SR, Kales SN, et al. An Official American Thoracic Society Statement: the importance of healthy sleep. Recommendations and future priorities. Am J Respir Crit Care Med. 2015;191(12):1450-1458. doi:10.1164/rccm.201504-0767ST

20. Federation ID. The IDF consensus worldwide definition of the metabolic syndrome; 2006. Available from: https://www.idf.org/ouractivities/advocacy-awareness/resources-and-tools/60:idfconsensusworldwide-definitionof-the-metabolic-syndrome.html. Accessed June 26, 2021.

21. Luo H, Li J, Zhang Q, et al. Obesity and the onset of depressive symptoms among middle-aged and older adults in China: evidence from the CHARLS. BMC Public Health. 2018;18(1):909. doi:10.1186/s12889-018-5834-6

22. Consultation We. Appropriate body-mass index for Asian populations and its implications for policy and intervention strategies. Lancet. 2004;363(9403):157-163. doi:10.1016/S0140-6736(03)15268-3

23. China country assessment report on ageing and health [press release]; World Health Organization; 2015. Available from: https:/www.who. int/ageing/publications/china-country-assessment/en/. Accessed June 26, 2021.
24. Hausler N, Marques-Vidal P, Haba-Rubio J, Heinzer R. Does sleep predict next-day napping or does napping influence same-day nocturnal sleep? Results of a population-based ecological momentary assessment study. Sleep Med. 2019;61:31-36. doi:10.1016/j. sleep.2019.04.014

25. Jing R, Barath D, Zhang H, Chen J, Fang H. Changes in sleep duration associated with retirement transitions: the role of naps. J Sleep Res. 2020;29(6):e12975. doi:10.1111/jsr.12975

26. Campbell SS, Stanchina MD, Schlang JR, Murphy PJ. Effects of a month-long napping regimen in older individuals. $\mathrm{J}$ Am Geriatr Soc. 2011;59(2):224-232. doi:10.1111/j.1532-5415.2010.03264.x

27. Hirshkowitz M, Whiton K, Albert SM, et al. National Sleep Foundation's sleep time duration recommendations: methodology and results summary. Sleep Health. 2015;1(1):40-43. doi:10.1016/j. sleh.2014.12.010

28. Faraut B, Nakib S, Drogou C, et al. Napping reverses the salivary interleukin-6 and urinary norepinephrine changes induced by sleep restriction. $J$ Clin Endocrinol Metab. 2015;100(3):E416-426. doi:10.1210/jc.2014-2566

29. Song Q, Liu X, Wang X, Wu S. Age- and gender-specific associations between sleep duration and incident hypertension in a Chinese population: the Kailuan study. J Hum Hypertens. 2016;30 (8):503-507. doi:10.1038/jhh.2015.118

30. Wu X, Sun Y, Niu K, et al. Association of self-reported sleep duration and hypertension: results of a Chinese prospective cohort study. Clin Exp Hypertens. 2016;38(6):514-519. doi:10.3109/ 10641963.2016.1163367

31. Guo J, Fei Y, Li J, Zhang L, Luo Q, Chen G. Gender- and agespecific associations between sleep duration and prevalent hypertension in middle-aged and elderly Chinese: a cross-sectional study from CHARLS 2011-2012. BMJ Open. 2016;6(9):e011770. doi:10.1136/ bmjopen-2016-011770

32. Borbely AA, Rusterholz T, Achermann P. Three decades of continuous wrist-activity recording: analysis of sleep duration. J Sleep Res. 2017;26(2):188-194. doi:10.1111/jsr.12492

33. Jackson CL, Patel SR, Jackson WB 2nd, Lutsey PL, Redline S. Agreement between self-reported and objectively measured sleep duration among white, black, Hispanic, and Chinese adults in the United States: multi-Ethnic Study of Atherosclerosis. Sleep. 2018;41 (6):1-12. doi:10.1093/sleep/zsy057

34. Al Lawati I, Zadjali F, Al-Abri MA. Agreement analysis of sleep patterns between self-reported questionnaires and actigraphy in adults. Sleep Breath. 2021. doi:10.1007/s11325-021-02296-1
Nature and Science of Sleep

\section{Publish your work in this journal}

Nature and Science of Sleep is an international, peer-reviewed, open access journal covering all aspects of sleep science and sleep medicine, including the neurophysiology and functions of sleep, the genetics of sleep, sleep and society, biological rhythms, dreaming, sleep disorders and therapy, and strategies to optimize healthy sleep.
The manuscript management system is completely online and includes a very quick and fair peer-review system, which is all easy to use. Visit http://www.dovepress.com/testimonials.php to read real quotes from published authors. 